\title{
Laparoscopic Adjustable Gastric Banded Plication (LAGBP): The Next Big Thing?
}

\author{
Kirubakaran Malapan • Ayham Ghinagow • \\ Andrea Ooi Se $\cdot$ Anirudh Vij $\cdot$ Po-Chih Chang $\cdot$ \\ Xiao-Yan Chen $\cdot$ Chih Kun Huang
}

Published online: 31 May 2013

(c) Springer Science + Business Media New York 2013

\begin{abstract}
Bariatric surgery comprising various restrictive, malabsorptive and combined procedures is an effective modality in treating morbid obesity. Laparoscopic adjustable gastric banded plication (LAGBP) is a novel restrictive bariatric procedure which was introduced in 2009. LAGBP may provide comparable results to laparoscopic sleeve gastrectomy at 2 years in terms of complications, \% excess weight loss (EWL) and co morbidity resolution. Modification in technique to a standardized LAGBP procedure which includes preserving right gastroepiploic vessels and standard plication volume facilitates in safety and feasibility of this procedure. The EWL at 6 months is $46.3 \%$ (range 29.38-73.35). LAGBP has been proven to be an effective bariatric surgery in the mid-term and may become a popular and cost effective technique.
\end{abstract}

Keywords Laparoscopic surgery - Bariatric surgery · Gastric banding · Sleeve gastrectomy · Gastric plication . Adjustable gastric banded plication - Laparoscopic adjustable gastric banded plication (LAGBP)

\section{Introduction}

Bariatric surgery is the most effective treatment for morbid obesity, producing durable weight loss, improvement or

K. Malapan - A. Ghinagow - A. O. Se - A. Vij · P.-C. Chang ·

C. K. Huang $(\bowtie)$

Department of Bariatric and Metabolic International Surgery

Centre, E-Da Hospital, No 1, E-Da Rd., Jiaosu Village,

Yan-Chau District, Kaohsiung City 82445, Taiwan

e-mail:dr.ckhuang@hotmail.com

X.-Y. Chen · C. K. Huang

The First Affiliated Hospital of Guangzhou Medical University,

Guangzhou 510182, China remission of comorbid conditions and longer life [1]. It can be broadly classified as restrictive, malabsorptive and combined. The gold standard bariatric procedure in many countries is the Laparoscopic Roux-En-Y Gastric Bypass (LRYGB) which is a combined restrictive and malabsorptive procedure [2]. Other malabsorptive procedures include biliopancreatic diversion (BPD) and biliopancreatic diversion with duodenal switch (BPD-DS). Though efficient in weight loss, these patients incur higher incidence of nutritional deficiencies and surgical complications like anastomotic leaks, stomal stenosis or bowel obstruction $[3,4]$.

Laparoscopic adjustable gastric banding (LAGB) has been a popular bariatric procedure because of its safety, efficacy, durability and adjustability [5]. However, in a long term prospective study of LAGB, Schouten et al. [6॰] reported $44 \%$ of their patients required re-operation or conversion to another bariatric procedure during follow up. Moreover, the band needs frequent adjustments, requiring multiple visits incurring cost and lifelong follow up not welcomed by patients [7]. Laparoscopic sleeve gastrectomy (LSG) is another restrictive procedure that is gaining popularity in Asia [8••]. However, the irreversible nature of the procedure and its associated complications have made it unacceptable to some patients. Weight regain and de novo gastroesophageal reflux (GERD) symptoms were reported, which appeared between the 3rd year and 6th year following surgery [9].

Talebpour and Amoli developed the laparoscopic total gastric vertical plication (LGCP) as an alternative restrictive procedure to LSG which minimized its potentially serious complications such as bleeding and staple line leaks. The procedure was also cheaper with minimal nutritional deficiencies. The estimated weight loss was $61 \%$ at 12 months, $60 \%$ at 24 months and $57 \%$ at 
36 months with acceptable morbidities [10]. Subsequently, authors have demonstrated that this operation was feasible and safe with comparable early results to other types of conventional procedures $[11,12]$.

As with other restrictive procedures, the possibility of postoperative weight regain due to the plicated gastric tube dilating remains debatable. We introduced a novel combination procedure involving both gastric banding and plication, which we termed Laparoscopic adjustable gastric banded plication (LAGBP) in 2009 [13••]. A combination of these two restrictive procedures could complement each other to increase weight loss, improve compliance and prevent weight regain [14••]. In a case matched comparative study with LSG, LAGBP showed similar results at 2 years in terms of complications and comorbidity resolution. The LSG appeared to be superior to LAGBP after 1 year in terms of $\%$ EWL, however at 2 years the \% EWL between the two procedures were comparable [15•]. The LAGBP could, therefore, be an alternative to LSG if a patient desires a restrictive and potentially reversible procedure.

This procedure is still in its infancy and lacks standardization in the operative technique compared with other more established bariatric procedures. Standardization may improve the efficacy of the procedure and lower the complication rates while ensuring reproducible results.

\section{Current Techniques}

Our initial technique of LAGBP was to place the adjustable gastric band first and then begin the plication below the band until the distal antrum using 2 layers of 2-0 Ethibond (Ethicon, Inc., Somerville, NJ, USA) sutures [13••]. Brethauer et al. [16] reported performing the plication in two layers with 2-0 polypropylene sutures, commencing just below the angle of His and continuing distally to within $4 \mathrm{~cm}$ of the pylorus. Similarly, Ramos et al., performed imbrication of the greater curvature over a 32-F bougie with 2-0 Ethibond (Ethicon, Inc., Somerville, NJ, USA) sutures for the 1st layer and 2-0 Prolene (Ethicon, Inc., Somerville, NJ, USA) for the 2nd layer.

Until June 2012, we had performed LAGBP in 111 morbidly obese patients with an average age of 32.2 years (range 18-60) using this technique (unpublished data). The mean pre-operative body mass index (BMI) was $39.2 \mathrm{~kg} /$ $\mathrm{m}^{2}$ (range 30.9-52.4). Ten patients $(9 \%)$ required readmission due to complications which included 1 trocar site hernia, 1 band leak, 1 gastric ulcer and 7 gastric fold herniations (GFH). GFH occurred more frequently in the first postoperative month $(4 / 7,57 \%)$ and at the fundus $(5 / 7$, $71 \%$ ). Mean excess weight loss (EWL) at 12 and 24 months after surgery was 52.1 and $62.6 \%$.
We hypothesized that the high incidence of GFH was multifactorial. The possible causes were placement of the adjustable band first and then performing the gastric plication, the wrong choice of suture material for plication and increased tissue oedema thereby weakening the plication sutures. Beginning July 2012 we made several changes in our surgical technique with respect to these possible factors and standardized the operative procedure. We conducted a prospective study to determine the safety and effectiveness of this standardized procedure.

This prospectively designed and retrospectively reviewed study was conducted between July 2012 and January 2013, with the approval of the E-DA Hospital institutional review board. We recruited all morbidly obese patients who underwent standardized LAGBP technique and provided written informed consent. Our inclusion criteria were based on the Asia Pacific Bariatric Surgery Group (APBSG) indication of bariatric surgery for Asians [17].

(1) Obese patients with a body mass index (BMI) $>37 \mathrm{~kg} / \mathrm{m}^{2}$;

(2) Obese patients with a BMI $>32 \mathrm{~kg} / \mathrm{m}^{2}$ in the presence of diabetes mellitus or another two significant comorbidities related to obesity;

(3) Obese patients who have been unable to lose or maintain weight loss through dietary or other forms of medical management;

(4) Patients whose age ranges from 18 to 65 years old.

We collected preoperative patient data, including the demographics, age, gender, height, weight, body mass index, and co-morbidities. Data regarding both intraoperative and postoperative complications, EWL and postoperative hospital stay were also recorded and analyzed. Complications were classified into major complications which were life threatening or required surgical intervention and minor complications which were managed conservatively but still required admission. Data were presented as mean standard deviation for continuous variables and frequency percentages for categorical variables. Continuous data were statistically analyzed using the independent $t$ test, and categorical data were analyzed using the Chi square test or Fisher's exact test. All tests were two-tailed and results were considered statistically significant if $P<0.05$. Statistical analyses were performed using the software package PASW, version 18, for Windows (SAS Institute, Cary, NC).

\section{Operative Procedure}

All surgical procedures took place under general anesthesia with the patient in supine position with their arms extended 
laterally in reverse trendelenburg position. The surgeon stood on the patient's right side and the assistant to the left. Closed pneumo-peritoneum was achieved and we used a five port technique. Trocar placement was as follows (A) one 12-mm trocar to the right of the umbilicus for the $30^{\circ}$ laparoscope; (B) one 15-mm trocar in the umbilicus for inserting the band and for the surgeon's right hand; $(\mathrm{C})$ one 5 -mm trocar in the upper right quadrant (URQ) in the midclavicular line for the surgeon's left hand; (D) one 5-mm trocar below the xiphoid process, slightly to the left of the falciform ligament for the self-retaining Nathanson liver retractor and (E) one 5-mm trocar in the patients upper left quadrant (ULQ) in the ant-axillary line for the assistant's instrument (Fig. 1).

In the standardized technique we began our dissection of the greater omentum using the Harmonic Scalpel (Ethicon Endo-Surgery, Cincinnati, Ohio) or Ligasure TM Vessel Ligation System (Covidien, Mansfield, MA) $3 \mathrm{~cm}$ from the pylorus and $1-2 \mathrm{~cm}$ from the greater curvature edge to preserve the right gastroepiploiec vessels (Fig. 2). Dissection was then continued up to the angle of His exposing the left crus of the diaphragm. The posterior gastric wall and gastropancreatic adhesions were released to facilitate in plicating the stomach.

We performed the stomach plication based on the individual patient's stomach size. Standardized measurement from the lesser curve to the greater curve $x \mathrm{~cm}$ was taken to determine the gastric plication formula (GPF). $(x \mathrm{~cm}+1) / 2$ (Fig. 3 and 4) The plication was performed using interrupted 2-0 Ethibond sutures (Excel Ethicon, StStevens-Woluwe, Belgium) at intervals of $2 \mathrm{~cm}$ to fix the shape of the gastric plication in a single layer along the greater curvature (Fig. 5). We commenced the plication from the fundus (just below the angle of His) and continued distally to the distal antrum. This was subsequently reinforced with continuous seromuscular suturing, using Polypropylene 2-0 suture (Ethicon, Inc., Somerville, NJ, USA) (Fig. 6).

The adjustable gastric band (REALIZE) was then placed using the pars flaccida technique. We did not perform gastro-gastric fixation for the band relying instead on the plication to prevent slippage. The reservoir port was fixed to the anterior rectus sheath near the umbilicus with 3-0 Polypropelene sutures (Ethicon, Inc., Somerville, NJ, USA) (Fig. 7). Skin was closed with subcuticular sutures.

Patients were given oral sips of water 4-6 h after the surgery. Oral proton pump inhibitors (PPIs), dexamethasone and tropisetron hydrochloride (Novoban) were prescribed for 1-2 days following surgery. Patients were discharged if the vital signs were normal and if vomiting was not observed after commencing liquid diet. Oral PPIs were prescribed for 1 month following surgery.

Patients adhered to a customized liquid diet for 1 week followed by pureed diet for another week. Semi-solid diet was commenced for 2 weeks followed by a progressive return to solid food in a stepwise fashion, depending on patient tolerance. Follow-up visits were scheduled for 1 week and $1,3,6,12,18$, and 24 months in the postoperative period. Adjustment of the gastric band was performed after 3 months during the clinic visit. Upper GI endoscopy was performed after 1 year for surveillance.

\section{Results}

Forty patients were included in the study of which 27 patients $(67.5 \%)$ were female and 13 patients $(32.5 \%)$ were male (ratio 1:1.9). The mean age was 33.4 years (range 19-55) and the mean pre operative BMI was $39.1 \mathrm{~kg} / \mathrm{m}^{2}$ (range 30.90-52.44). The obesity associated co-morbidities

Fig. 1 Port insertion sites

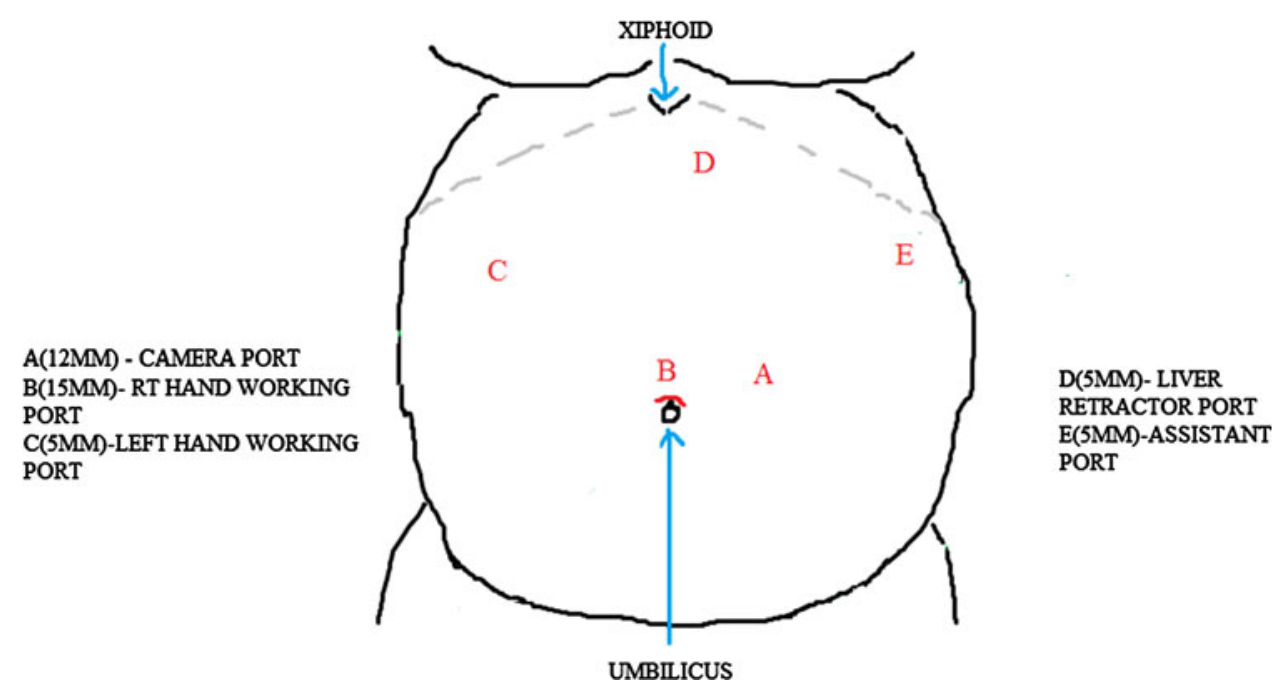




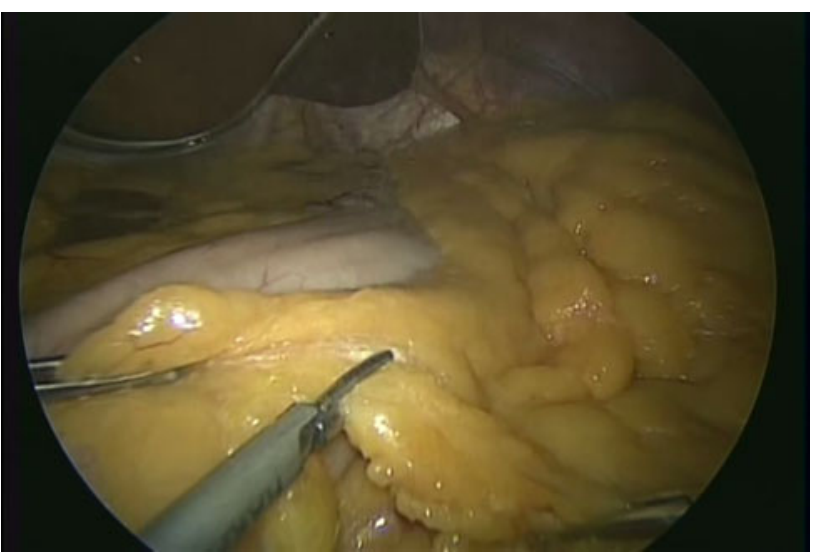

Fig. 2 Preservation of gastroepiploeic vessels during gastrolysis

were hyperlipidemia $30(78.9 \%)$, hyperuricemia 8 (21.1\%), hypertension $10(26.3 \%)$, Type 2 DM, 4 (10.5\%) and fatty liver $35(92.1 \%)$ (Table 1$)$.

The mean total operative time was 108.1 min (range 41-189) of which, $98.7 \mathrm{~min}$ (range 37-184) was the mean plication time and $9.2 \mathrm{~min}$ (range 3-32) was the mean banding time. There were no intra-operative complications. The mean duration of hospital stay of stay was 1.9 days (range 1-3) (Table 2).

Two patients were admitted within the 1 st week of surgery for dehydration following episodes of vomiting and abdominal pain. Both patients were admitted, managed conservatively and discharged after 2 days. There were neither major complications nor mortality in our series of patients. The mean $\%$ EWL at 3 months was $36.5 \%$ (range 24.07-57.32) and at 6 months was $46.4 \%$ (range 29.38-73.35).

\section{Discussion}

LAGB has been a popular bariatric procedure due to acceptable weight loss, resolution of co-morbidities and low operative morbidity [18]. Patients failure to attend follow-up appointments and the persistent consumption of calorie-dense food after the procedure often led to poor weight loss results [19]. In a 8 year follow up following LAGB, Suter et al. [20] reported a nearly $40 \%$ 5-year failure rate, and a $43 \%$ 7-year success rate $(\mathrm{EWL}>$ $50 \%$ ). This data suggests that LAGB should no longer be considered as the procedure of choice for obesity.

LSG is a popular stand-alone bariatric procedure that has demonstrated similar weight loss and resolution of comorbidities to that of duodenal switch or Roux-en-Y gastric bypass [21]. Despite this, irreversibility of the procedure and surgical complications such as hemorrhage, leaks, abscesses, strictures, gastroesophageal reflux disease and mortality are a concern for the patient and surgeon [22]. Based on current published literature, LSG has a risk/ benefit profile that lies between LAGB and LRYGB [23]. LGCP is a new and potentially effective restrictive procedure with EWL of 61 and $60 \%$ at 1 and 2 years, respectively [10]. However, patients have the possibility of weight gain after 1 year due to dilatation of the plicated stomach [12].

LAGBP was introduced as a novel surgical procedure in 2009 that could serve as a salvage procedure for patients who experienced band failure [14••]. Besides being able to overcome the complications and irreversibility of LSG, LAGBP could improve the weight loss of LGCP alone, by adjustment of the band. We reported that it was a safe, technically feasible, and reversible procedure that resulted

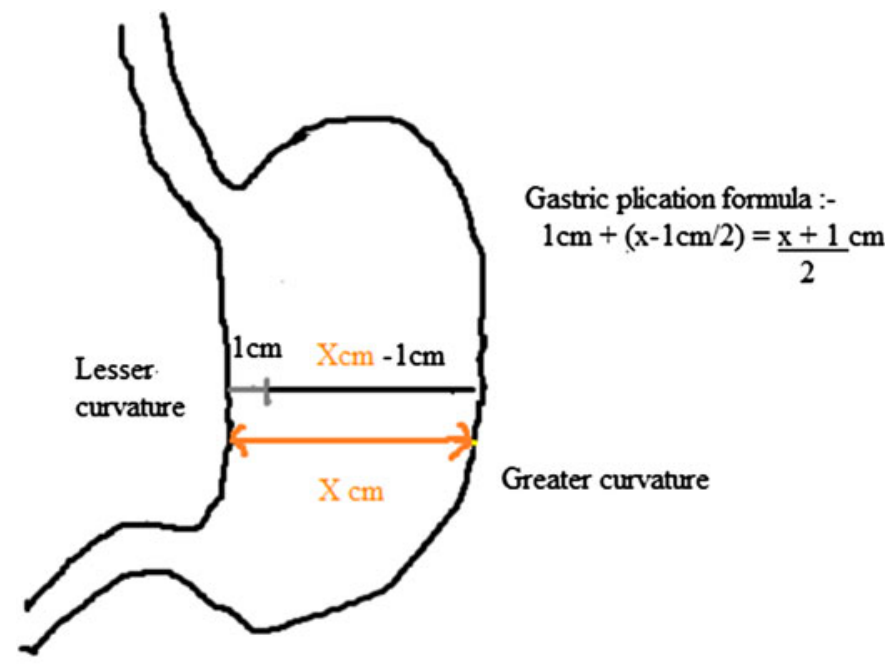

TRANSVERSE VIEW

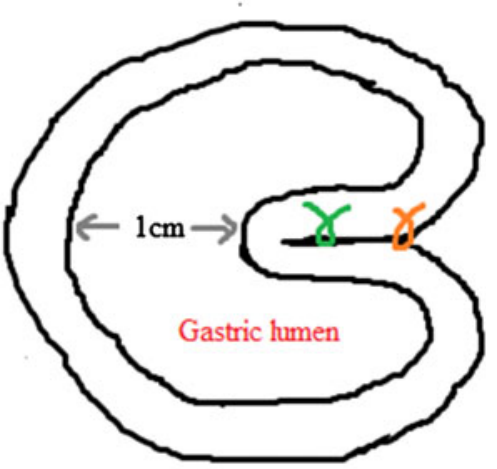

1st layer interupted ethibond $2 / 0$ 2nd layer continuous prolene $2 / 0$

Fig. 3 Gastric plication diagram 


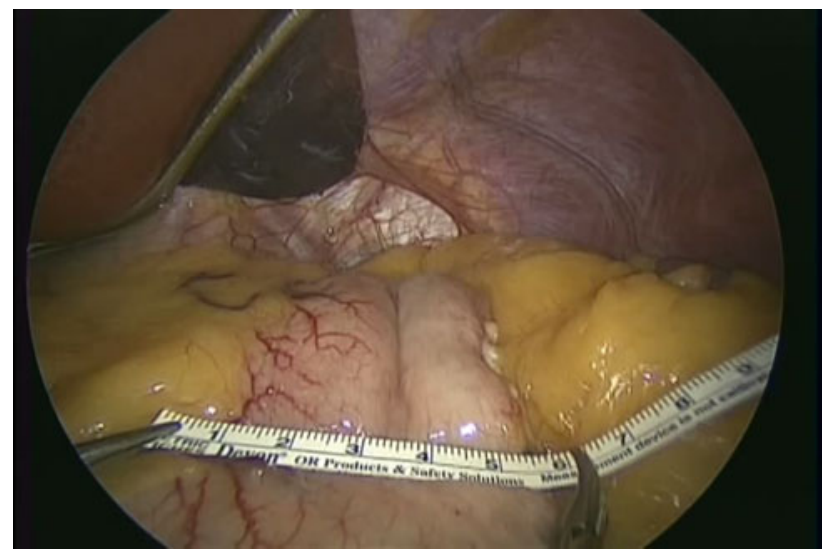

Fig. 4 Taking measurements and applying the gastric plication formula

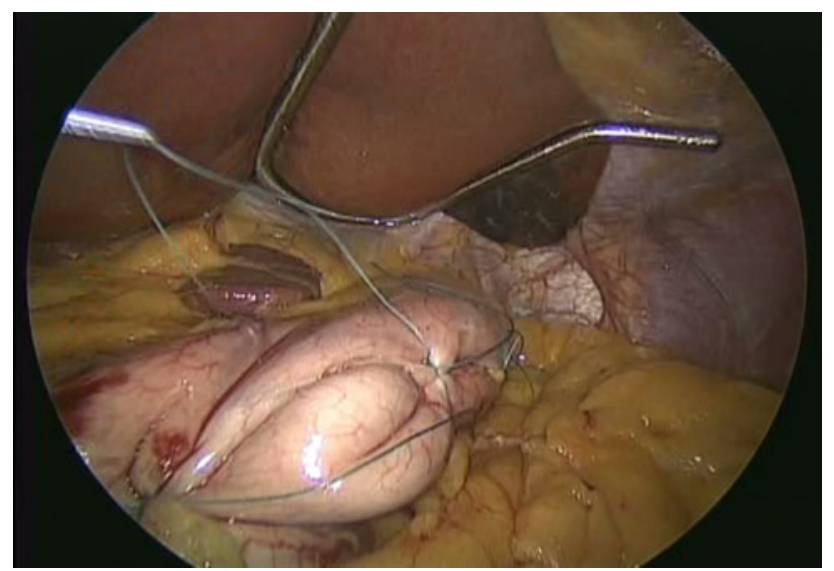

Fig. 5 First layer plication with interrupted Ethibond 2-0

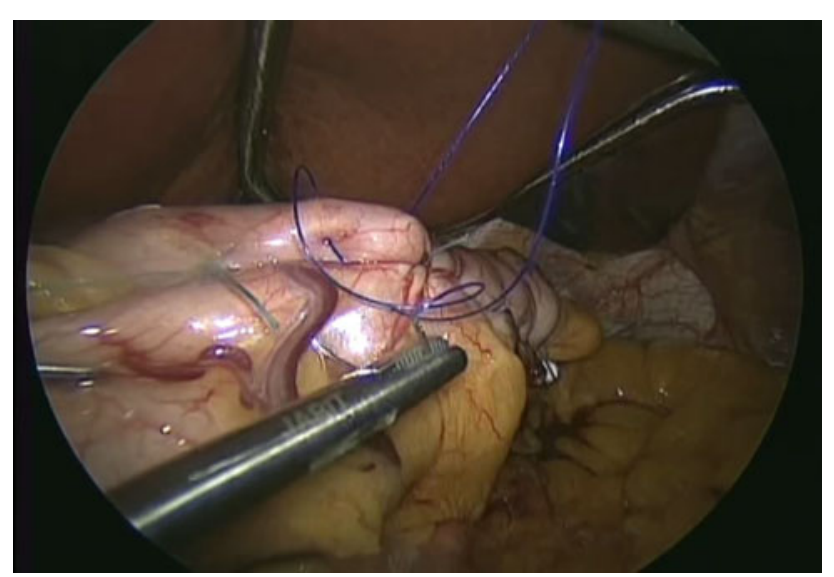

Fig. 6 Second layer plication with continuous Prolene 2-0

in acceptable weight loss during the early postoperative period $[13 \bullet \bullet, 24 \bullet \cdot$. In a case matched comparative study between LAGBP and LSG, LAGBP showed similar results at 2 years in terms of complications and co morbidity

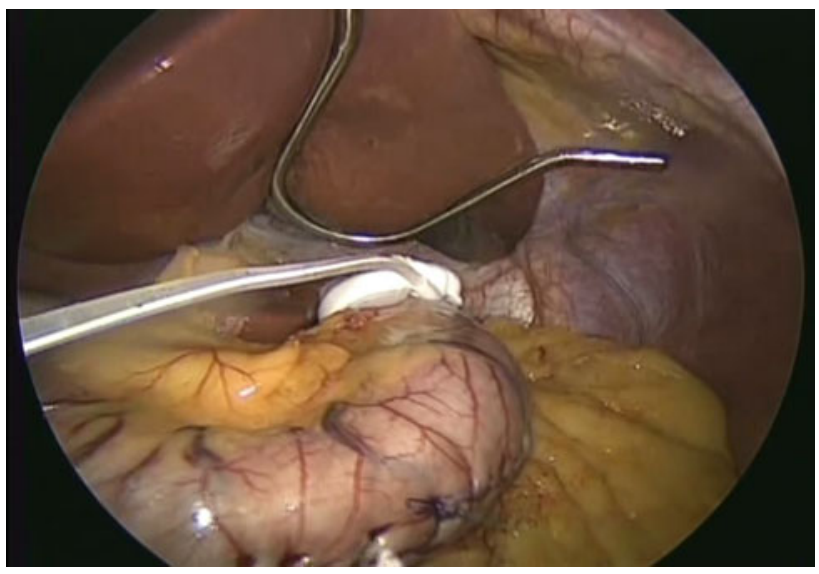

Fig. 7 Completed LAGBP

Table 1 Gender, co-morbidities and complications

\begin{tabular}{lcc}
\hline Variable & Number & Percentage \\
\hline Male & 13 & 32.5 \\
Female & 27 & 67.5 \\
Hyperlipidemia & 30 & 78.9 \\
Hyperuricemia & 8 & 21.1 \\
Hypertension & 10 & 26.3 \\
Type 2 DM & 4 & 10.5 \\
Fatty liver & 35 & 92.1 \\
Major complications & 0 & 0 \\
Minor complications & 2 & 5 \\
\hline
\end{tabular}

Table 2 Patient Demography and Surgical outcomes for standardized LAGBP procedure

\begin{tabular}{lcl}
\hline Variable $(n=40)$ & Mean \pm SD & Range \\
\hline Age $($ years $)$ & $33.45 \pm 9.93$ & $(19-55)$ \\
Height $(\mathrm{cm})$ & $166.92 \pm 7.78$ & $(149-184.9)$ \\
Weight $(\mathrm{kg})$ & $109.89 \pm 20.45$ & $(78.9-160.4)$ \\
BMI $\left(\mathrm{kg} / \mathrm{m}^{2}\right)$ & $39.17 \pm 4.84$ & $(30.90-52.44)$ \\
Total operation time $(\mathrm{min})$ & $108.15 \pm 40.20$ & $(41-189)$ \\
Plication time $(\mathrm{min})$ & $98.70 \pm 38.30$ & $(37-184)$ \\
Banding time $(\min )$ & $9.20 \pm 6.61$ & $(3-32)$ \\
Length of stay $(\mathrm{days})$ & $1.9 \pm 0.53$ & $(1-3)$ \\
Follow up EWL $(\%)$ & $36.92 \pm 13.82$ & $(24.07-57.32)$ \\
$\quad$ at 3 months $(n=21)$ & & \\
Follow up EWL $(\%)$ & $46.39 \pm 15.02$ & $(29.38-73.35)$ \\
$\quad$ at 6 months $(n=14$ & & \\
\hline
\end{tabular}

resolution. LSG appeared to be superior to LAGBP after 1 year in terms of \% EWL, however, at 2 years the \% EWL between both procedures were comparable presumably due to the synergistic effect of the band [15•]. LAGBP could, 
therefore, be an alternative to LSG if a patient desires a restrictive and reversible procedure without anatomical changes.

Currently, there is no standardization in the gastric plication technique. Several authors have reported variations in their technique, bougie size and choice of suture material used $[11,12,16]$. The incidence of GFH in our series was higher compared to that reported in a systematic review of gastric plication for morbid obesity [25••]. A possible cause for GFH was our initial technique of placing the band first and then plicating the stomach, which resulted in inadequate plication adjacent to the band. Reversing the order by performing the gastric plication first, ensures proper plication of the fundus and a more uniformly placed plication line.

Gastric plication relies on serosal adhesion formation within the fold to maintain its durability. Replacing Ethibond 2-0 (Excel Ethicon, St-Stevens-Woluwe, Belgium) with Polypropylene 2-0 suture (Ethicon, Inc., Somerville, NJ, USA) for the 2nd layer made suturing easier with less tissue trauma and uniform tension on the plication line. Individualizing the amount of stomach to be plicated for each patient based on the GPF avoided unnecessary excess plication. Adding a constant distance of $1 \mathrm{~cm}$ to the gastric diameter (transverse) prevents oedema and venous congestion within the intra-luminal fold which could cause obliteration of the gastric lumen and gastric obstruction.

Another possible factor for increasing tissue oedema, thereby weakening the plication line, is devascularization of the greater curvature vessels. Here, we recommend preserving the right gastroepiploic vessels to maintain venous return. This maneuver prevents worsening oedema of the gastric wall and intraluminal fold. We have yet to encounter any more complications related to GFH after making the above changes in our surgical technique.

A recent systematic review reported, 25 (8\%) out of 307 patients who underwent gastric plication for morbid obesity developed complications. Nausea and vomiting was reported in all these studies ranging from mild to moderate. It usually resolved within 1-2 weeks and most of the cases did not require readmission and were managed with antiemetics and prokinetics [25••]. In our series, the most frequent complaint was vomiting in the early postoperative period, which mostly resolved within $48 \mathrm{~h}$. However, two patients needed re-admission following discharge for intravenous hydration and antiemetics. The possible mechanisms were outlined as above. We did not routinely insert a nasogastric tube after surgery. Patients can be treated with generous intravenous hydration, administration of PPIs and prokinetic agents to relieve their symptoms. It is very important for patients to adhere to the post operative diet regime to prevent post operative nausea and vomiting. Rupture of the sutures and gastric perforation could occur due to resuming solid diet too early after surgery, before serosal adhesion is complete.

A previously performed long-term follow-up study of 591 LAGB patients revealed that the complication rate was about $23.3 \%$ and included band failure, slippage, erosion and infection [26]. There were no band related complications seen in this study. LAGB is considered to have the lowest complication rate among all contemporary bariatric surgery procedures. However, patients with a gastric band must be followed up closely and usually require multiple adjustments of the band [27]. Complications following LAGBP such as band slippage and erosion could be avoided as the band does not require fixation sutures. We also anticipate lesser frequency of band adjustments thereby decreasing the risk of infection around the injection port. It remains to be seen if longer follow up will reveal other band related problems.

LAGBP would readily be accepted by patients and bariatric surgeons alike. Reversibility, accessibility to the whole stomach and minimal vitamin supplementation gives added value to this remarkable procedure. It could also be considered as a salvage procedure for patients who have experienced gastric band failure.

\section{Conclusions}

LAGBP has been proven to be an effective bariatric procedure in the mid-term and will become popular soon, after adopting this standardized technique. However, long term follow-up is necessary to confirm these findings and for it to be an efficient stand alone bariatric procedure.

Disclosure Kirubakaran Malapan, Ayham Ghinagow, Andrea Ooi Se, Anirudh Vij, Po-Chih Chang, Xiao-Yan Chen and Chih Kun Huang declare that they have no conflict of interest.

Compliance with Ethics Guidelines This article does not contain any studies with human or animal subjects performed by any of the authors.

\section{References}

Papers of particular interest, published recently, have been highlighted as:

- Of importance

•- Of major importance

1. Guideline for clinical application of laparoscopic bariatric surgery, SAGES Guidelines Committee, Society of American Gastrointestinal Endoscopic Surgeons (SAGES) 6/2008.

2. Jacobsen HJ, Bergland A, Raeder J, et al. High-volume bariatric surgery in a single center: safety, quality, cost-efficacy and 
teaching aspects in 2,000 consecutive cases. Obes Surg. 2012;22(1):158-66.

3. Rabkin Robert A, Rabkin John M, Metcalf Barbara, et al. Laparoscopic technique for performing duodenal switch with gastric reduction. Obes Surg. 2003;13(2):263-8.

4. Aills Linda, Blankenship Jeanne, Buffington Cynthia, et al. ASMBS allied health nutritional guidelines for the surgical weight loss patient. Surg Obes Relat Dis. 2008;4(5 Suppl): S73-108.

5. Burton PR, Brown WA. The mechanism of weight loss with laparoscopic adjustable gastric banding: induction of satiety not restriction. Int J Obes. 2011;35(Suppl 3):S26-30.

6. - Schouten R, Wiryasaputra DC, van Dielen FMH. Long-term results of bariatric restrictive procedures: a prospective study. Obes Surg. 2010;20(12):1617-26. A long term prospective study on LAGB showing a $44 \%$ reoperation or reintervention rate.

7. Shen R, Dugay G, Rajaram K, et al. Impact of patient follow up on weight loss after bariatric surgery. Obes Surg. 2004;14(4): 514-9.

8. • Lomanto D, Lee WJ, Goel R, et al. Bariatric surgery in Asia in the last 5 years (2005-2009). Obes Surg. 2012;22(3):502-6. A perspective on the bariatric procedures performed in Asia showing the decline in band procedures and rise in sleeve gastrectomy.

9. Himpens J, Dobbeleir J, Peeters G. Long-term results of laparoscopic sleeve gastrectomy for obesity. Ann Surg. 2010;252(2): 319-24.

10. Talebpour M, Amoli BS. Laparoscopic total gastric vertical plication in morbid obesity. J Laparoendosc Adv Surg Tech A. 2007;17(6):793-8.

11. Ramos A, Neto MG, Galvao M, et al. Laparoscopic greater curvature plication: initial results of an alternative restrictive bariatric procedure. Obes Surg. 2010;20(7):913-8.

12. Mui WLM, Lee DWH, Lam KKY, et al. Laparoscopic greater curve plication in Asia: initial experience. Obes Surg. 2013;23(2): 179-83.

13. • Chih-Kun Huang MD, Chi-Hsien Lo MD, Asim Shabbir MD. Novel bariatric technology: laparoscopic adjustable gastric banded plication: technique and preliminary results. Surg Obes Relat Dis. 2012;8(1):41-5. The first published paper on the analysis of 26 patients who underwent LAGBP procedure.

14. •• Huang CK, Asim S, Lo CH. Augmenting weight loss after laparoscopic adjustable gastric banding by laparoscopic gastric plication, Surg Obes Relat Dis. 2011;7(2):235-6. The first case report demonstrating gastric plication in augmenting weight loss following $L A G B$.
15. - Huang CK, Chhabra N, Goel R, et al. Laparoscopic adjustable gastric banded plication: a case-matched comparative study with laparoscopic sleeve gastrectomy. Obes Surg. 2013. doi: 10.1007/s11695-013-0951-x. A comparative study showing that the LAGBP procedure is as efficacious with acceptable results as a popular restrictive bariatric procedure.

16. Brethauer SA, Harris JL, Kroh M, et al. Laparoscopic gastric plication for treatment of severe obesity. Surg Obes Relat Dis. 2011;7(1):15-22.

17. Lee WJ, Wang W. Bariatric surgery: Asia-Pacific perspective. Obes Surg. 2005;15(6):751-7.

18. Chapman AE, Kiroff G, Game P, et al. Laparoscopic adjustable gastric banding in the treatment of obesity: a systematic literature review. Surgery. 2004;135(3):326-51.

19. Miller KA. Evolution of gastric band implantation and port fixation techniques. Surg Obes Relat Dis. 2008;4(3 Suppl):S22-30.

20. Suter M, Calmes JM, Paroz A, Giusti V. A 10-year experience with laparoscopic gastric banding for morbid obesity: high longterm complication and failure rates. Obes Surg. 2006;16(7): 829-35.

21. Bohdjalian A, Langer FB, Shakeri-Leidenmuhler S, et al. Sleeve gastrectomy as sole and definitive bariatric procedure: 5-year results for weight loss and ghrelin. Obes Surg. 2010;20(5): 535-40.

22. Dietel M, Gagner M, Erickson AL, et al. Third international summit: current status of sleeve gastrectomy. Surg Obes Relat Dis. 2011;7(6):749-59.

23. American Society for Metabolic and Bariatric Surgery; updated position statement on sleeve gastrectomy as a bariatric procedure revised 10/28/2011.

24. •• Goel R, Chang P-C, Huang C-K. Reversal of gastric plication after laparoscopic adjustable gastric banded plication. Surg Obes Relat Dis. 2013;9(1):e14-5. A paper demonstrating that it is possible to reverse gastric plication and thereby proving that $\angle A G B P$ is indeed a reversible, restrictive bariatric procedure.

25. - Abdelbaki TN, Huang C-K, Ramos A, et al. Gastric plication for morbid obesity: a systematic review. Obes Surg. 2012;22(10):1633-9. A systematic review on gastric plication showing the various procedures done by different surgeons and lack of standardization.

26. Biagini J, Karam L. Ten years experience with laparoscopic adjustable gastric banding. Obes Surg. 2008;18(5):573-7.

27. Buchwald $\mathrm{H}$, Avidor $\mathrm{Y}$, Braunwald $\mathrm{E}$, et al. Bariatric surgery: a systematic review and meta-analysis. JAMA. 2004;292(14): $1724-37$. 